\title{
Individual based modeling of angiogenesis in three dimensions on a graphics processing unit
}

\author{
L. Parsonson \& L. Bai \\ School of Computer Science, University of Nottingham, UK
}

\begin{abstract}
Angiogenesis, the formation of de novo blood vessels, has been implicated in a long list of human disorders, including cancer, diabetes, and in recent years, neurodegenerative diseases, a list that continues to grow. Study of the formation of microvasculature, therefore, has important implications in diagnosis and treatment of diseases. This paper describes how we combine a CPUbased Cellular Potts model of sprouting angiogenesis in three dimensions with medical imaging techniques and graphics processing unit (GPU) accelerated fluid dynamics equations to create an individual-based angiogenesis simulation. The use of GPU, optimized for fast, highly parallel mathematical operations, provides an increase in simulation speed and balances resource requirements across hardware. Specifically, micro-CT scans of resin cast rat cerebral vasculature are segmented to remove reconstruction artifacts and are imported to instantiate nascent endothelial cells in a homogeneous three-dimensional grid that represents the area over which the simulation is performed. A growth factor source is added, and simulation of steady production and diffusion of the vascular endothelial growth factor (VEGF) is performed on the GPU using the NVidia Compute Unified Device Architecture (CUDA) programming platform. Motion of individual endothelial cells is then tracked over the lifetime of the simulation towards the source of growth factor, incorporating both sprouting and anastamosis events.
\end{abstract}

\section{Introduction}

The microvasculature, that is the smallest vessels within the human body, is implied in more and more diseases, including, but not limited to, cancer, diabetes and rheumetoid arthritis. In cancer, for example, the growth of tumors is reliant upon securing a nutrient source, which amounts to a blood supply, and this is 
achieved when the tumor begins to secrete angiogenic factors which in turn cause the migration of endothelial cells and the formation of new vasculature $[1,2]$. This has further implications in the form of metastasis, where cancer cells detach from a tumor and enter the bloodstream, causing the spread of the disease across the body when they invade otherwise healthy tissue elsewhere. Recent research in Alzheimer's Disease (AD) has also highlighted the role of vascular structure in terms of early diagnosis of the disease, especially the microvasculature which are responsible for the exchange of nutrients across the blood-brain barrier [3]. This has raised the possibility that abnormalities in the vascular structure may be an early indication of dementia; vascular abnormalities result in impaired cerebral blood flow and down regulation of the amyloid- $\beta$ clearance receptor, and thus leading to increased risk of AD.

Angiogenesis, the formation of de novo blood vessels from existing vasculature through sprouting, branching, and restructuring, can be categorized into two forms; that of vessel restructuring as found in embryonic angiogenesis (also referred to as vasculogenesis) and that of new vessel growth such as found during wound healing (neoangiogenesis). Angiogenesis is triggered by a variety of factors [4]. Of key interest is when hypoxia in cells causes the release of various growth factors, most notable of which is Vascular Endothelial Growth Factor A (VEGFA) [5]. Initially, formation of new blood vessels is triggered by a breakdown of the native vessels [6]. This is followed by migration of endothelial cells towards the source of a growth factor. During this process, the extracellular matrix (ECM) is broken down by proteins secreted by endothelial cells, allowing the new vessels to bind to the matrix. Movement of endothelial tip cells is a partially stochastic process (discussed in more detail later) and thus anastomosis (looping) of vessels can occur, as well as splitting of the emerging vessel.

\section{Related work}

In the past fifteen or so years, considerable effort has been made in the development of mathematical models of angiogenesis. Mathematical models rely on the phenomenon of hypoxic regions of tissue producing growth factors which diffuse across the tissue towards existing vessels, causing endothelial cell migration and the creation of de novo vasculature. This is often combined with hydrodynamic models to assess the effectiveness of the vasculature, and direct remodeling efforts. While such attempts to 'grow' simulated vascular networks in silico have been the most popular approach, some efforts have been made to generate vasculature following tree-based or fractal methods. This has, however, had limited success. Tree based models lack the looping (anastomoses) required.

Anderson and Chaplain [7] developed a model of tumor induced angiogenesis (often referred to as tumorigenesis) based on motion of migrating endothelial tip cells in two-dimensions governed by three functions described by partial differential equations; the chemotactic function, or tendency to climb a growth factor gradient; the haptotactic function, relating to the requirement of fibronectin; and endothelial cell migration in two dimensions. This system of equations was 
then solved using finite difference methods to provide a discretization of the model which could be applied to a regular grid describing a unit square (i.e. a numerical domain of $[0,1] \times[0,1])$ representing an area of width $2 \mathrm{~mm}$. The square was divided into a grid of $200 \times 200$ cells, offering a resolution of $0.005 \mathrm{~mm}$ per unit. This system was expanded to include random branching events and looping due to fusion of cells which met (anastomosis).

This led the way for further improvements and modifications of the scheme such as using a hexagonal gird [8], maturation [9], and application of fluid dynamics. Godde and Kurz [8] developed a model which works on a hexagonal grid and considers shear-stress and pressure dependent systems. There model showed that shear-stress dependent modeling led to a more homogeneous distribution of capillaries connecting terminal arterial and venous vessels than that of pressure dependent modeling. Owen et al. [10] developed a multi-scale model of angiogenesis and vascular remodeling which accounts for vessel pruning due to low wall shear stress amongst other factors. Linninger and Vaicaitis [11] showed some results of a vasculature generation algorithm which displays similarity to scanned vasculature of human subjects, but give little in the way of implementation details.

Watson et al. [12] developed a model of vascular development in the murine retinal plexus during neonatal development, involving modeling development of the astrocyte network which precedes vasculogenesis in neonatal mice. This approach employs similar modeling techniques to those outlined by Anderson and Chaplain in 1998 [7] in tracking individual astrocyte and endothelial tip cells. However, this model adds increased complexity through remodeling of vasculature by simulating blood flow through the emerging vascular network. This involves simulation of blood flow through the network, and delivery of oxygen to surrounding tissue as a result of this, followed by pruning of the capillary network in light of relevant criteria.

\section{GPU based angiogenesis modeling}

The modelling method described here is a three-dimensional model. Unlike the two-dimensional model developed by Anderson and Chaplain [7], the extra dimension introduces computational complexity, which has led to the GPU implementation of the fluid dynamics to provide a mechanism for growth factor diffusion within the bounds of the model. As a whole, the method is a combination of a Cellular Potts model (CPM), which models cell movement, and partial differential equations (PDEs) which are used to govern the release and subsequent diffusion of chemical attractants. CPM is a well known lattice-based computational modeling technique which represents unit sections of an arbitrary space as cells on a grid, updating them based on a set of probabilistic rules. Within a CPM an object being modeled can occupy multiple cells in the grid. During the initialization step of the simulation, a stack of images acquired from a microCT scan are imported. The images used are of resin casts of rat cerebral vasculature, taken at a resolution of $15 \mu \mathrm{m}$, approximately the size of an individual cell. Thus, 
pixels in the scans are likened to cells within the original vasculature. Images are thresholded, and individual pixels are inserted into the domain of the simulation as nascent endothelial cells. Before the simulation begins, the domain is scanned for cells which are entirely enclosed - that is, they have neighbors in all six principal directions. Any cell found to match these conditions is flagged for deletion before the simulation begins.

\subsection{Endothelial tip cell movement}

Typically, models of angiogenesis describe the growth of new vessels from existing vasculature via tracking the path of migrating endothelial cells. The movement of these cells is initiated by disruption of the basement membrane which surrounds the endothelium of existing vessels, thus allowing for free movement of endothelial cells. Movement is then tracked under the assumption of endothelial cells migrating towards the source of the growth factor. The equation for endothelial tip cell motion is as follows:

$$
\frac{\partial n}{\partial t}=D_{\mathrm{n}} \nabla^{2} n-\chi_{0} \nabla \cdot(n \nabla c)-\rho_{0} \nabla \cdot(n \nabla f)
$$

where $n$ is the density of endothelial cells, $D_{\mathrm{n}}$ is the random motility coefficient, $\chi$ the chemotactic function, $c$ the growth factor density, $\rho$ the haptotactic function, and $f$ the fibronectin density. Movement of endothelial tip cells is derived from a discretized form of equation 1. Discretization is achieved by applying the finite difference method [13] which yields three equations, relating to each of the components of equation (1). These three equations relate to the three characteristics influencing cell migration; movement up a gradient of growth factor, movement up a gradient of fibronectin, and a random walk component.

Endothelial cells are modeled as agents on the CPU. Each agent is modeled individually, and thus is aware only of itself and its immediate environment. As a simplification measure in terms of program structure, a grid (three-dimensional array) of numerical values is maintained as part of the environment, indicating whether a particular location within the simulated section is occupied by a cell or vessel segment. The numerical value is an identifier of the cell which filled this space - in the case of a vessel segment, the identity of the originating cell is given. These values are used to prevent a cell looping into itself, causing early termination of the simulation which sometimes occurs due to random movement.

When a cell moves, the direction in which it can move is constrained to those empty cells which are in front of the cell. These cells are identified by taking the dot product of the vector from the cell's current position and the vector of the direction in which the EC last moved. If the result is above a threshold value (in this case 0.5 , implying an angle of less than 90 degrees between the two vectors), the cell is a viable target direction in which to move. Enabling this constraint removes the possibility of ECs moving in a direction which would be physically impossible. Deciding which direction an endothelial cell moves is performed by distributing probabilities across the set of cells available for movement. This 
involves finding both the cell in the direction of continued movement and the cell in the direction of the overall chemotactic gradient. The chemotactic gradient is calculated by summing the differences of the growth factor densities across the local environment of the EC:

$$
\sum_{\substack{-1<i<1 \\-1<j<1 \\-1<k<1}}|\chi(i, j, k)-\chi(0,0,0)| \times(i, j, k)
$$

where $\chi(i, j, k)$ is the growth factor density at the displacement $(i, j, k)$ from the ECs location. This yields a vector which is normalized to give $\hat{\chi}$, describing the direction of the growth factor gradient at the location of the EC. This vector is used to identify the cell which is in the direction of the chemotactic gradient. The continued movement cell is simply identified by repeating the last displacement.

These two cells are given weighted probabilities as defined by constants $\chi$ and $\psi$. The remaining cells are assigned an equal portion of $1-\chi-\psi$. This represents the random portion of the movement equation, and is more apparent when the values for the chemotactic and haptotactic biases are low (see figure 2).

After assigning these probabilities, they are combined to form a distribution which ranges from 0.0 to 1.0. A random number is generated in this range, and the cell assigned the range in which the number falls is allocated as the direction in which the EC moves.

\subsection{Vessel formation}

As the density of growth factor increases, so does the possibility of branching, that is, splitting into two vessels. However, in order for a vessel to branch it must be of a threshold age. When branching occurs, this original vessel sprout is considered to be complete, and two new sprouts produced, thus the age is reset. In this case, a new agent is created and added to the environment at the location of the sprouting; this location is randomized in the locale of the original endothelial cell, and follows the constraint of occurring perpendicular to the last direction in which the original cell moved. This is assigned to the cell as the direction of last movement, and thus encourages the sprout, initially at least, to move away from the parent vessel.

Anastomosis occurs when a migrating endothelial tip cell comes into contact with another sprout. When contact occurs, the migrating tip cell is essentially removed from the agent simulation, and the sprout becomes fixed. However, if an EC comes into contact with it's own vessel sprout, anastomosis is ignored; this is primarily to prevent a terminal anastomosis, that is early termination of the simulation due to random motion causing an EC to loop onto itself and stop movement. This does not, however, remove the possibility of a new sprout, as described above, moving into its parent sprout and anatomizing, however, due to the uptake of growth factor by occupied spaces and the diffusion element, this is unlikely to occur. 


\subsection{Physical constraints and boundary conditions}

Every model is limited by the size of the simulation, although constraints vary over different models. Models of angiogenesis tend to be limited to a square or cuboid environment, with no-flux boundary conditions imposed, such as in the model developed by Anderson and Chaplain [7]. Lemon et al. [14] modeled angiogenesis within a virtual pore, analogous to an hourglass shape, to simulate angiogenesis within a porous tissue engineering scaffold [15]. Perfahl et al. [16] employed differing sets of boundary conditions, initially no-flux as employed by Anderson and Chaplain [7] but also periodic boundary conditions.

In this model, boundary conditions were set to be no-flux, that is, nothing can enter or escape the confines of the simulated volume; fluids or agents. This has the effect of simplifying the model to some extent, removing the possibility of external influences. A constrained volume is required to prevent the simulation growing uncontrollably and arbitrarily increasing memory and processor requirements. Similarly, the size of the simulation is limited to an area approximately $1 \mathrm{~mm}^{3}$, which maintains reasonable memory requirements and execution speed.

\subsection{Growth factor modeling on the GPU}

One of the key elements indicated in studies of vascular growth are the various growth factors [4], chemicals which encourage the migration and growth of endothelial cells, resulting in neovascularization. These chemical factors are subject to diffusion and decay, and thus must be produced in large enough quantities to create a sustained gradient in order to cause endothelial cell migration. A focus of many studies is vascular endothelial growth factor (VEGF), which tends to be produced under hypoxic circumstances.

\subsubsection{Modeling on the GPU}

Diffusion of growth factors can be modeled using standard diffusion reaction equation, defined as follows:

$$
\nabla^{2} P-\frac{1}{D} \frac{\partial P}{\partial t}=0
$$

where $P$ is the fluid density and $D$ the diffusion coefficient. In a discrete model, this can approximated using the finite difference method as described in [13]. This element of a simulation lends itself to a GPU-based implementation, since it involves iteration of every element of an entire grid (or mesh, in three dimensions), and thus is well suited to the parallelism available on a GPU. The 3D grid structure of the discretized fluid continuum and the largely homogeneous nature of the implicit update function (4):

$$
\rho_{i, j, k}^{*}=\frac{\Delta x^{2} \rho_{i, j, k}+a}{\Delta x^{2}+b}
$$


where $\rho_{i, j, k}$ is the fluid density at grid location $(i, j, k), \Delta x$ is the grid unit size, $a$ is the product of diffusion coefficients $d$ and $\rho$ neighboring $(i, j, k)$, and $b$ the total of the diffusion constants $\mathrm{d}$, lend themselves well to hardware designed to process large amounts of linear data in a massively parallel fashion. GPU devices devote more of their hardware real-estate to raw data processing, rather than reserving a large amount of architecture for memory caching and flow control, as with conventional CPUs. That is, they are best suited to data-parallel applications linear arrays of data with each element processed via the same program - focusing on high arithmetic intensity rather than memory operations. As a result, they are not a general purpose solution to computational problems. Tasks requiring a large amount of random memory access or logic branching are more suited to conventional CPUs. For example, the linked list structure of the level set narrow band and the sense-decision-action logic of the agents are more naturally suited to the memory and logic capabilities of the CPU. However, as mentioned, the linear nature of an inhomogeneous diffusion algorithm fits perfectly with the GPGPU (General Purpose Graphics Processing Unit) model. In this work, NVIDIA's CUDA (Compute Unified Device Architecture) is used. For the sake of generality, the specifics of the implementation will be restricted to a general overview.

\subsubsection{Memory structure}

To implement the 3D inhomogeneous diffusion equation using the GPU, several data arrays are required on both the host (the CPU)-side and the device (the GPU)side. For straightforward integration with the deformable surface, the size of the fluid's discretization lattices are matched to the size of the level set lattice $\phi$, allowing for a one-to-one correspondence in queries and updates. The memory requirements are divided as follows:

- An array for the fluid lattice on the host, $\rho^{\text {host }}$.

- An array for storing the diffusion coefficient lattice on the host, $D^{\text {host }}$.

- An array for storing the fluid lattice on the device, $\rho^{\text {device }}$.

- An array for storing the iteratively computed fluid lattice on the device, $\rho^{*}$. This is double-buffered with $\rho^{\text {device }}$, so that the previous value of $\rho$ becomes the initial guess for the Gauss-Seidel iterative algorithm on the next time step.

- A texture for storing the diffusion coefficient lattice on the device, $D^{\text {device }}$. Texture memory is read-only on the device side, but has extra caching allowing for much faster memory fetches than the alternative global memory used for the other device-side data arrays.

Each time step of the main simulation $\rho^{\text {device }}$ must be downloaded to $\rho^{\text {host }}$. Once $\rho^{\text {host }}$ and $D^{\text {host }}$ are updated, they are both uploaded to $\rho^{\text {device }}$ and $D^{\text {device }}$, respectively. The bandwidth for download/upload between the main system memory and the GPU's memory is relatively low, but this latency is more than made up for by the increase in computation speed. 


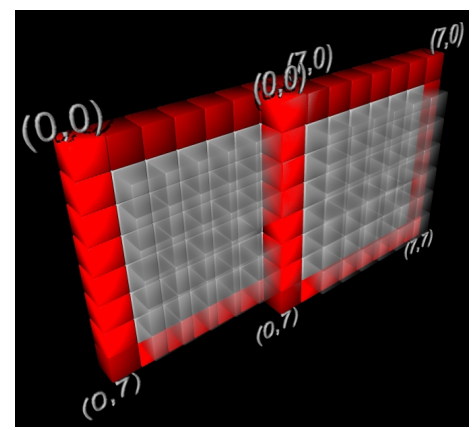

Figure 1: Illustration of CUDA inhomogeneous diffusion implementation thread block layout, showing two $8 \times 8$ blocks side by side. Each block handles a $2 \mathrm{D}$ chunk of data, with each thread (cube) handling a single lattice node. Blocks march along the $\mathrm{z}$-axis of the lattice, with each thread updating $\rho^{*}$ at its respective lattice location using (4). Each central thread (white cube) fetches three lattice nodes from global memory into the shared memory cache: if a thread indexes to $(x, y, z)$ then the lattice values at points $(x, y, z-1),(x, y, z)$ and $(x, y, z+1)$ are fetched. The edge threads (red cubes) only fetch the value at $(x, y, z)$ into shared memory. Once the data are fetched, the edge threads of the block terminate, since spatial derivatives require the values from $\pm x, \pm y$ and $\pm z$, which the edge threads do not have. Thus there is an overlap between thread blocks in order to compute the values at these edge elements.
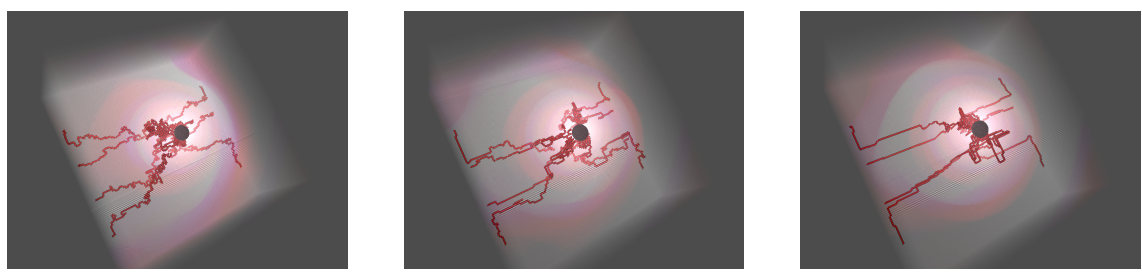

Figure 2: Increasing the chemotactic bias produces straighter vessels, whereas decreasing produces more random, tortuous results. Shown here are results with bias values of 0.4 (left), 0.5 (center) and 0.6 (right).

\section{Results and discussion}

This model utilizes GPU and CPU techniques, taking advantage of parallelism by modeling diffusion on the GPU and employing an agent-based method of tracking individual endothelial cells on the CPU. Each step of a cell agent's movement is said to represent one unit of new vasculature. As vasculature grows, diffusion constants are modified to reflect the difference in the milieu of the simulation growth factors are less able to diffuse into areas occupied by new vasculature. 
Figure 3 shows the growth of such a network over time. Movement is essentially randomized, but biased towards the direction of the previous move made and up the gradient of growth factor. Figure 2 shows how the chemotactic bias affects the shape of the vasculature. With a lower bias, the movement of endothelial tip cells is more randomized, and thus longer, more tortuous vessels are produced. With a higher bias, straighter, albeit less realistic vessels are generated.

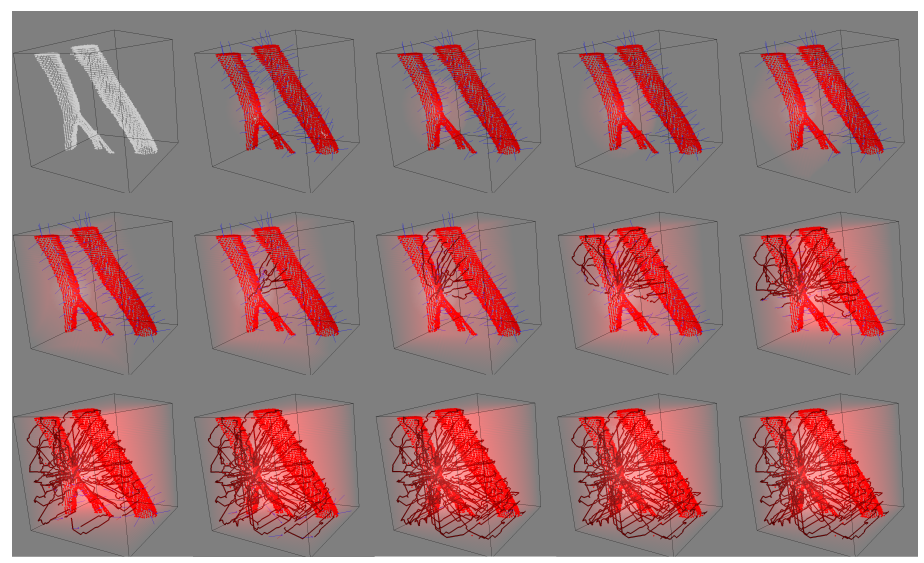

Figure 3: Example of growth of a capillary network over time. Initially, cells are nascent. Introduction of a growth factor gradient (pink) activates cells (blue cells) around which is an exclusion region (red cells). As the growth factor density reaches a threshold level, activated cells migrate towards the source of the gradient. New vessels are drawn differently to highlight topographical features.

Continuation of this work could take a variety of paths, however current efforts are being focused on the simulation of blood flow through growing vascular networks, and the associated pruning aspects, as well as the effect of oxygen delivery on growth factor production.

\section{References}

[1] Folkman, J., Angiogenesis in cancer, vascular, rheumatoid and other disease, Nature Medicine, Volume 1, Number 1, Nature Publishing Group (1995)

[2] Travasso, R., Poiré, E., Castro, M., Rodriguez-Manzaneque, J., HernándezMachado, A., Tumor Angiogenesis and Vascular Patterning: A Mathematical Model, PloS ONE 6(5): e19989. doi:10.1371/journal.pone.0019989 (2011)

[3] Iadecola, C., Rescuing troubled vessels in Alzheimer disease, Nature Medicine, Volume 11, Number 9, September 2005 (2005)

[4] Folkman, J., Klagsbrun, M., Angiogenic Factors, Science, Volume 235 (1987) 
[5] Yancopoulos, G., Davis, S., Gale, N., Rudge, J., Wiegand, S., Holash, J., Vascular-specific growth factors and blood vessel formation, Nature, vol 407, 14 September (2000)

[6] Rahman, R., Smith, S., Rahman, C., Grundy, R., Antiangiogenic Therapy and Mechanisms of Tumor Resistance in Malignant Glioma, Journal of Oncology, Volume 2010, Article 251231 (2010)

[7] Anderson, A., Chaplain, M., Continuous and Discrete Mathematical Models of Tumor-induced Angiogeneisis, Bulletin of Mathematical Biology, 60, 857900 (1998)

[8] Godde, R., Kurz, H., Structural and Biophysical Simulation of Angiogenesis and Vascular Remodelling, Developmental Dynamics, 220, 387-301 (2001)

[9] Arakelyan, L., Vainstein, V., Agur, Z., A computer algorithm describing the process of vessel formation and maturation and its use for predicting the effects of anti-angiogenic and anti-maturation therapy on vascular tumor growth, Angiogenesis 5, 203-214 (2002)

[10] Owen, M., Alarcon, T., Maini, P., Byrne, H., Angiogenesis and vascular remodelling in normal and cancerous tissues, Journal of Mathematical Biology, 58, 689-721 (2009)

[11] Linninger, A., Vaicaitis, N., Computational Modeling of Cerebral Vasculature, Computational Modeling of Cerebral Vasculature, Laboratory for Product and Process Design, Department of Bioengineering, University of Illinois at Chicago (2011)

[12] Watson, M., McDougall, S., Chaplain, M., Devlin, A., Mitchell, C., Dynamics of angiogenesis during murine retinal development: a coupled in vivo and in silico study, J. R. Soc. Interface, doi:10.1098/rsif.2012.0067, March 2012 (2012)

[13] Mitchell, A., Griffiths, D., The finite difference method in partial differential equations, Chichester: Wiley (1980)

[14] Lemon, G., Howard, D., Rose, F., King, J., Individual-based modelling of angiogenesis inside three-dimensional porous biomaterials, Elsevier, BioSystems, 103, 372-383 (2011)

[15] Novosel, E., Kleinhause., C., Kluger, P., Vascularization is the key challege in tissue engineering, Advanced Drug Delivery Reviews, 63, 300-311, Elsevier (2011)

[16] Perfahl, H., Byrne, H., Tingan, C., Estrella, V., Alarcón, T., Lapin, A., Gatenby, R., Gillies, R., Lloyd, M., Maini, P., Reuss, M., Owen, M., Multiscale Modelling of Vascular Tumour Growth in 3D: The Roles of Domain Size and Boundary Conditions, PloS ONE, Volume 6, Issue 4, April 2011 (2011) 